\title{
Association of Catheter Ablation for Atrial Fibrillation with Mortality and Stroke: a systematic review and meta-analysis
}

\author{
Sérgio Barra ${ }^{1}$, Jakub Baran ${ }^{1,2}$, Kumar Narayanan ${ }^{3,4}$, Serge Boveda $^{5}$, Simon Fynn ${ }^{1}$, \\ Patrick Heck $^{1}$, Andrew Grace ${ }^{1}$, Sharad Agarwal ${ }^{1}$, João Primo ${ }^{6}$, Eloi Marijon ${ }^{3,7,8}$, Rui \\ Providência 9
}

${ }^{1}$ Cardiology Department, Papworth Hospital NHS Foundation Trust, Cambridge, UK

2 Division of Clinical Electrophysiology, Department of Cardiology, Grochowski Hospital, Postgraduate Medical School, Warsaw, Poland

${ }^{3}$ Paris Cardiovascular Research Center, Paris, France

${ }^{4}$ Cardiology Department, MaxCure Hospitals, Hyderabad, India

${ }^{5}$ Cardiology Department, Clinique Pasteur, Toulouse, France

${ }^{6}$ Cardiology Department, V. N. Gaia Hospital Center, V. N. Gaia, Portugal

${ }^{7}$ Cardiology Department, European Georges Pompidou Hospital, Paris, France

${ }^{8}$ Paris Descartes University, Paris, France

${ }^{9}$ Barts Heart Centre, Barts Health NHS Trust, London, UK

\section{Corresponding author:}

Sérgio Nuno Craveiro Barra

Papworth Hospital NHS Foundation Trust, Papworth Everard, Cambridge CB23 3RE, UK E-mail address: sergioncbarra@gmail.com

Word count: 3446

Financial support: None

Conflicts of interest relevant to this study: None 
All authors take responsibility for all aspects of the reliability and freedom from bias of the data presented and their discussed interpretation. 


\section{ABSTRACT (286 words)}

Background: Maintenance of sinus rhythm has been associated with lower mortality, but whether atrial fibrillation (AF) ablation per se benefits hard outcomes such as mortality and stroke is still debated.

Objective: To determine whether AF ablation is associated with a reduction in all-cause mortality and stroke compared with medical therapy alone.

Methods: Literature search looking for both randomized and observational studies comparing AF catheter ablation vs. medical management. Data pooled using random-effects. Risk ratios (RR) with 95\% confidence intervals (CI) used as a measure of treatment effect. The primary and secondary outcomes were all-cause mortality and occurrence of cerebrovascular events during follow-up, respectively.

Results: Thirty studies were eligible for inclusion, comprising 78,966 patients $(25,129$ receiving AF ablation and 53,837 on medical treatment) and 233,990 patient-years of followup. The pooled data of studies revealed that ablation was associated with lower risk of allcause mortality: $5.7 \%$ vs. $17.9 \%$; $R=0.44,95 \% \mathrm{CI} 0.32-0.62, \mathrm{p}<0.001$. In a sensitivity analysis by study design, a survival benefit of AF ablation was seen in randomized studies, with no heterogeneity (mortality risk $4.2 \%$ vs. $8.9 \%$; $\mathrm{RR}=0.55,95 \% \mathrm{CI} 0.39-0.79, \mathrm{p}=0.001$, $\left.\mathrm{I}^{2}=0 \%\right)$, and also in observational studies, but with marked heterogeneity ( $6.1 \%$ vs. $18.3 \%$; $\left.\mathrm{RR}=0.39,95 \% \mathrm{CI} 0.26-0.59, \mathrm{p}<0.001, \mathrm{I}^{2}=95 \%\right)$. The mortality benefit in randomized studies was mainly driven by trials performed in patients with LV dysfunction and heart failure. The pooled risk of a cerebrovascular event was lower in patients receiving AF ablation (2.3\% vs. 5.5\%; RR=0.57, 95\%CI 0.46-0.70, $\mathrm{p}<0.001, \mathrm{I}^{2}=62 \%$ ), but no difference was seen in randomized trials $\left(2.2 \%\right.$ vs. $2.1 \%$; $\left.\mathrm{RR}=0.94,95 \% \mathrm{CI} 0.46-1.94, \mathrm{p}=0.87, \mathrm{I}^{2}=0 \%\right)$. 
Conclusions: Ablation of atrial fibrillation associates with a survival benefit compared with medical treatment alone, although evidence is restricted to the setting of heart failure and left ventricular systolic dysfunction.

Key-words: Ablation; atrial fibrillation; mortality; stroke; meta-analysis.

\title{
ABBREVIATIONS
}

\author{
AF - Atrial fibrillation \\ CI - Confidence interval \\ $\mathbf{E F}$ - Ejection fraction \\ LV - Left ventricular \\ $\mathbf{R R}$ - Risk ratio
}




\section{INTRODUCTION}

Atrial fibrillation (AF) is the most common sustained cardiac arrhythmia and its prevalence is on the rise worldwide due to aging of the population and the increased survival from conditions often associated with $\mathrm{AF}^{1}$. Approximately $1 \%$ of the general population is estimated to have AF, with a prevalence of up to $10 \%$ among elderly patients ${ }^{2}$. Atrial fibrillation is a major cause of stroke and is associated with increased cardiovascular and overall mortality ${ }^{3}$.

In randomized trials, catheter ablation has been shown to effectively reduce $\mathrm{AF}$ recurrence and burden and improve quality of life when compared with antiarrhythmic therapy ${ }^{4,5}$, and therefore it has become a widely accepted treatment to restore and help maintain sinus rhythm in symptomatic patients who have failed antiarrhythmic therapy (class I, level of evidence $A)^{3}$. In the AFFIRM trial, which did not include patients undergoing catheter ablation, patients remaining in sinus rhythm for longer durations had reduced mortality ${ }^{6}$. However, the overall comparison of rhythm-control vs. patients receiving rate control alone in this study failed to show any significant survival benefit or reduction in the risk of stroke which was attributed to the adverse effects of antiarrhythmic drugs ${ }^{7}$. Therefore, if AF catheter ablation can maintain sinus rhythm with higher efficacy and lower rate of complications than antiarrhythmic drugs, it has the potential to improve patient outcomes with respect to stroke and cardiovascular mortality. Thus far, results in this regard from different observational ${ }^{8-11}$ and randomized trials ${ }^{12-15}$ have been inconclusive and somewhat contradictory.

We therefore conducted an up-to-date systematic review and meta-analysis of randomized and observational studies aiming to assess the impact of catheter ablation on the risk of mortality and cerebrovascular events (stroke/transient ischaemic attacks). 


\section{METHODS}

\section{I - Data sources, Literature Search and Eligibility Criteria}

We performed searches on MEDLINE (via PubMED), EMBASE, clinicaltrials.gov and COCHRANE databases (from inception to August 31, 2017) using the following search string: ((“ablation” AND “atrial fibrillation”) AND (“stroke” OR "thromboembolism” OR “mortality” OR “death”)). Reference lists of all accessed full-text articles were searched for sources of potentially relevant information and experts in the field were contacted about further potentially eligible studies. Authors of full-text papers were also contacted by email to retrieve additional information. Manual searches were performed to identify studies presented at Hotline sessions of major cardiovascular conferences.

Only longitudinal studies performed in humans, written in English and with a minimum follow-up duration of 6 months were considered for inclusion. Registries, observational studies and randomized trials were considered eligible for analysis. The population, intervention, comparison and outcome (PICO) approach was used ${ }^{16}$. The population of interest included patients with atrial fibrillation and the intervention was catheter ablation or medical treatment \pm electrical cardioversion alone. Comparisons were performed between patients receiving catheter ablation for atrial fibrillation (pulmonary vein isolation \pm additional substrate ablation) vs. those who did not receive an ablation. The primary outcome was total all-cause mortality, evaluated at the longest follow-up available. The secondary outcome was the occurrence of a cerebrovascular event (stroke or transient ischaemic attack) during follow-up. The methods sections of evaluated studies were reviewed to confirm the suitability and composition of the reported endpoint.

Two independent reviewers (SB and JB) screened all abstracts and titles to identify potentially eligible studies. The full text of all such studies was further evaluated to determine 
the final suitability of the study for inclusion in the review and meta-analysis. Agreement of both reviewers was required for decisions regarding inclusion or exclusion of studies.

\section{II - Validity/Quality Assessment}

This meta-analysis complies with the preferred reporting items of PRISMA for systematic reviews and meta-analyses ${ }^{17}$. Study quality was formally evaluated using the Delphi Consensus criteria for randomized controlled trials ${ }^{18}$ and a modified Newcastle-Ottawa Quality Assessment Scale for Cohort Studies ${ }^{19}$ by both reviewers (SB and JB). An agreement was mandatory for the final classification of studies.

\section{III - Data extraction and synthesis}

The following data were extracted for characterizing each patient sample in the selected studies, whenever available: mean age, sex, type of ablation, duration of AF prior to the ablation, type of AF (paroxysmal or persistent), treatment with antiarrhythmic drugs, heart failure severity, mean $\mathrm{CHA}_{2} \mathrm{DS}_{2}$-VASc score, mean left ventricular (LV) ejection fraction (EF) left atrial diameter, history of arterial hypertension, diabetes mellitus, coronary artery disease/previous myocardial infarction, cerebrovascular event and lung disease, and followup duration. A standardized form was used by the authors responsible for data collection and each of these performed this task in an independent fashion. The different forms were then compared to confirm the accuracy of the data and any discordance discussed with a separate author who was not originally involved in data collection.

Data were pooled using random-effects, according to the Mantel-Haenszel model, using the Comprehensive Meta-analysis v3 software. The risk ratio (RR) with respective 95\% confidence intervals (95\% CI) was used as a measurement of treatment effect. Pairwise comparisons were performed for the endpoints of total all-cause mortality and 
cerebrovascular events. For observational studies, outcome data obtained after propensity score matching was used whenever available to reduce the risk of treatment selection bias. A supplementary analysis was performed to assess the individual contribution of each study to the pooled estimate by recalculating the pooled RR after excluding that particular study.

Statistical heterogeneity on each outcome of interest was quantified using the $\mathrm{I}^{2}$ statistic, which describes the percentage of total variation across studies due to heterogeneity rather than chance. Values of $<25 \%, 25-50 \%$ and $>50 \%$ are by convention classified as low, moderate, and high degrees of heterogeneity, respectively.

Funnel plots were used for evaluating the presence of publication bias and traced for comparisons including more than 10 studies (minimum number for assuring the appropriateness of the method) (Higgins JPT, Green S (editors). Cochrane Handbook for Systematic Reviews of Interventions Version 5.1.0 [updated March 2011]. The Cochrane Collaboration, 2011. Available from: www.cochrane-handbook.org). We used the Trim and Fill adjustment for assessing the impact of any publication bias.

\section{IV - Subgroup Analyses and Meta-Regression}

Sensitivity analyses were performed to assess potential differences in clinical effectiveness between catheter ablation vs. medical treatment alone depending on study design: randomized vs. non-randomized. The sensitivity analysis of randomized trials was particularly important given the expected treatment selection bias in the observational studies. However, the sensitivity analysis of observational studies was still performed for two main reasons: firstly, this would provide a reasonable assessment of mortality and stroke rates in "real life" patients; secondly, it would illustrate the differences in mortality and stroke rates between patients included in randomized trials and those seen in daily clinical practice. These data may be useful when discussing treatment options with patients and managing their 
expectations. A further sensitivity analysis was performed after excluding observational studies which did not use propensity score matching. A meta-regression (using the Unrestricted ML method) was also performed for comparisons involving more than 10 studies for assessing the possible association of moderator variables with the primary endpoint. The following potential moderator variables were assessed: date of publication, age, sex, follow-up duration, previous history of hypertension, Diabetes Mellitus, coronary artery disease/myocardial infarction and cerebrovascular event, LV systolic function and left atrial diameter. In a meta-regression, the outcome variable is the effect estimate and the moderator variables are characteristics of studies that might influence the effect estimate. The regression coefficient obtained from a meta-regression analysis describes how the effect estimate changes with a unit increase in the moderator variable.

\section{RESULTS}

\section{Search results and study characteristics}

Out of a total of 2514 entries resulting from the initial literature search, 171 were retrieved for analysis of titles and abstracts and the full text version was accessed when deemed appropriate. Eighteen studies were considered adequate for inclusion in our meta-analysis on the basis of our inclusion criteria ${ }^{8-13,20-31}$. The literature search also retrieved four different meta-analyses from which 8 additional studies were found adequate ${ }^{14-16,32-37}$. One study comparing pulmonary vein isolation vs. atrioventricular node ablation plus biventricular pacemaker implantation was excluded as the non-AF ablation group was also submitted to invasive procedures, including ablation ${ }^{38}$. Manual searches provided 4 additional studies ${ }^{39-42}$, including a paper in press $^{41}$ and a randomized trial presented at a Hotline session of a major cardiovascular conference ${ }^{42}$ (and subsequently published). In summary, this meta-analysis 
included a total of $\mathbf{3 0}$ studies. Supplementary figure $\mathbf{1}$ illustrates the study selection process.

The design of selected trials and baseline data are summarized in table $\mathbf{1}$ and supplementary table 1. The final population for this meta-analysis included 78,966 patients (25,129 receiving catheter ablation and 53,837 receiving medical treatment alone) and 233,990 patient-years of follow-up (71,067 in patients receiving ablation and 161,922 in those not receiving ablation). Nineteen studies were randomized controlled trials ${ }^{9,12-15,25,28-}$ $37,40-42$, including 2,721 patients in total, whereas the remaining 11 were observational studies and/or registries $8,10,11,20-24,26,38,39$ and included 76,245 patients. Twenty-one studies were multi-centre ${ }^{8-15,20,23,24,26-30,32,34,37,41,42}$. Quality assessment of the included studies is shown in Supplementary Table 2. The overall study quality was good, as seven randomized controlled studies had $\geq 6$ Delphi criteria and 8 cohort studies had a Newcastle-Ottawa score of $\geq 7$.

Despite the fact that i) 19 studies were randomized trials ${ }^{9,12-15,25,28-37,40-42}$, and ii) approximately two thirds of outcome data related to patients retrieved from observational studies was obtained through propensity score or case-control matching $8,10,11,21,23,27,39$, treatment groups were not completely balanced at baseline when considering all studies. This was unequivocally due to the treatment selection bias expected in the observational studies, even when mitigated by the use of propensity score or case-control matching.

Supplementary table 1 describes the baseline characteristics of ablation and non-ablation groups included in each study. However, study groups were very well balanced in randomized studies, as shown in table 2.

\section{AF catheter ablation and outcomes}

The pooled data of studies revealed that AF patients undergoing ablation had significantly lower all-cause mortality rates compared with those who did not receive an ablation 
(supplementary figure 2): $5.7 \%$ vs. $17.9 \% ; \mathrm{RR}=0.44,95 \% \mathrm{CI} 0.32-0.62, \mathrm{p}<0.001$. The observed $\mathrm{I}^{2}$ values showed marked heterogeneity within this analysis $\left(\mathrm{I}^{2}=88 \%\right)$. In a sensitivity analysis by study design, a survival benefit of AF ablation was seen in randomized studies, with no heterogeneity (mortality $4.2 \%$ vs. $8.9 \%$; RR=0.55, 95\% CI 0.39-0.79, $\left.\mathrm{p}=0.001, \mathrm{I}^{2}=0 \%, \mathrm{NNT}=21\right)$ (Figure 1). Mortality was also seen to be significantly lower in the ablation arm in observational studies, but with marked heterogeneity $(6.1 \%$ vs. $18.3 \%$; $\mathrm{RR}=0.39,95 \% \mathrm{CI} 0.26-0.59, \mathrm{p}<0.001, \mathrm{I}^{2}=95 \%, \mathrm{NNT}=8$ ) (Figure 1). After excluding all observational studies which did not use propensity score or case-control matching, AF ablation patients still had lower all-cause mortality $(6.8 \%$ vs. $16.3 \%$; RR=0.48, 95\%CI 0.34 $\left.0.68, \mathrm{p}<0.001, \mathrm{I}^{2}=63 \%, \mathrm{NNT}=10.5\right)$. Funnel plots for the primary endpoint did not reveal any significant publication bias (supplementary figure 3).

The risk of a cerebrovascular event was overall lower in patients receiving an ablation (2.3\% vs. 5.5\%; RR=0.57, 95\%CI 0.46-0.70, p<0.001, $\left.\mathrm{I}^{2}=62 \%, \mathrm{NNT}=31\right)$ [supplementary figure 4], but the difference was restricted to observational data $(2.3 \%$ vs. $5.5 \%, \mathrm{RR}=0.54$, 95\% CI 0.43-0.68, $\mathrm{p}<0.001, \mathrm{I}^{2}=75 \%$ ). In randomized trials, no difference in stroke risk was seen $\left(2.2 \%\right.$ vs. $2.1 \%$; RR=0.94, 95\%CI 0.46-1.94, $\left.\mathrm{p}=0.87, \mathrm{I}^{2}=0 \%\right)$ (Figure 2); it is noteworthy that only 32 cerebrovascular events in total were seen during follow-up. Funnel plots for this secondary endpoint did not reveal any significant publication bias (supplementary figure 5).

Clinical periprocedural strokes, which were not included in our study endpoints, were reported in 9 patients subjected to ablation in randomized studies. Furthermore, there was one peri-procedural death. Data on peri-procedural strokes in observational data was very limited.

\section{Meta-regression and Moderator Variables}


The assessment of potential moderator variables through meta-regression revealed that the heterogeneity in the benefit of catheter ablation for the reduction of mortality was partly explained by differences in mean age, sex, history of hypertension, Diabetes Mellitus, coronary artery and cerebrovascular disease and year of study publication (supplementary table 3). For studies with higher mean patient age, catheter ablation was shown to be of more pronounced benefit in the reduction of all-cause death (supplementary figure 6). Also, the benefit of ablation was more pronounced in older (vs. more recent) studies and studies with higher percentage of female patients. Conversely, in studies with higher prevalence of Diabetes Mellitus, coronary artery and cerebrovascular disease the benefit was less pronounced.

Likewise, catheter ablation was of more pronounced benefit in the reduction of stroke in studies with higher mean patient age, a higher percentage of female patients and more prevalent cerebrovascular disease (supplementary table 3).

\section{DISCUSSION}

Although ablation of atrial fibrillation has historically been performed for symptom relief and improving quality of life, whether it provides survival benefit has been a matter of controversy. Our large, pooled analysis provides insights in this regard. In this meta-analysis, AF ablation was associated with lower mortality risk, which was noted in both randomized and observational studies. Patients who received ablation also had a lower risk of cerebrovascular events, but this effect was only seen in observational studies and therefore further adequately powered randomized trials addressing this endpoint are needed to clarify whether ablation can be of benefit for stroke reduction.

In the randomized studies, the pooled benefit was mostly attributable to two studies, namely the AATAC trial by Di Biase et al ${ }^{12}$ and the recently published Catheter Ablation 
versus Standard conventional Treatment in patients with Left ventricular dysfunction and Atrial Fibrillation (CASTLE-AF) Trial ${ }^{42}$. Both studies enrolled patients with heart failure and significant LV systolic dysfunction ${ }^{12,42}$, which is a special setting where AF ablation may be of particular benefit as noted in prior smaller studies. Successful AF ablation has been shown to lead to improvements in left ventricular function ${ }^{41,43,44}$, especially when sinus rhythm is maintained $^{45}$, which may have prognostic impact in heart failure patients. The very recent Catheter Ablation Versus Medical Rate control in Atrial Fibrillation and Systolic Dysfunction (CAMERA-MRI) study elegantly demonstrated that restoration of sinus rhythm with catheter ablation results in significant improvements in ventricular function in persistent AF patients with idiopathic cardiomyopathy, especially in the absence of ventricular fibrosis on cardiac $\mathrm{MR}^{40}$. Additionally, in AF patients with heart failure, antiarrhythmic drug therapy is limited to Amiodarone and Dofetilide, which often cause significant adverse effects, further augmenting the potential benefit of ablation in this scenario. Therefore, while it is difficult to be currently dogmatic about an overall mortality benefit of AF ablation in all-comers, a benefit among patients with LV dysfunction seems likely, in line with previous evidence.

The mortality benefit was also robustly noted in observational studies, even when restricted to studies with propensity matching, which lends some credence to the results, though even such matching strategies cannot completely eliminate bias inherent to observational studies. Although six of the observational studies achieved maximum rate according to the modified Newcastle-Ottawa Quality Assessment Scale, matched cohorts may still differ in regards to unmeasured or unknown variables. Patients submitted to ablation are in theory fitter and have a lower degree of comorbidity than those receiving medical treatment alone, as shown in this meta-analysis. As such, the extent of mortality benefit noted from observational studies, while encouraging, needs to be taken with caution. 
With regard to the outcome of stroke, a reduction was noted only with observational studies and the aforementioned limitations of observational design need to be borne in mind. No stroke reduction was noted in the pooled analysis of randomized trials. Any potential benefit of catheter ablation in the reduction of long-term stroke is possibly counterbalanced against the peri-procedural risk of stroke, which remains a significant complication of AF ablation but was not consistently reported in the observational studies. It has been suggested that successful ablation and restoration of sinus rhythm, although acutely associated with an increase in the risk of silent cerebral emboli, may subsequently decrease embolic burden over time ${ }^{46}$; however this hypothesis remains controversial, as recently shown by Ghanbari et $\mathrm{al}^{47}$, and the results from the present analysis do not lend strong support to the theory of stroke reduction. These observations notwithstanding, it should be highlighted that none of randomized studies were powered to show a benefit of ablation in the reduction of stroke risk.

Multiple studies have shown that catheter ablation is superior to medical treatment alone for the prevention of recurrent AF, morbidity reduction and improvement in quality of life $\mathrm{e}^{39,43,48}$. Moreover, it may seem logical that successful AF ablation will lead to a reduced risk of death and cerebrovascular event compared with antiarrhythmic drug therapy alone, as i) subjects with $\mathrm{AF}$ have markedly reduced survival compared to subjects without $\mathrm{AF}^{49}$ and higher risk of heart failure; ii) the burden of AF has been shown to associate with the risk of stroke ${ }^{50}$; iii) ablation is superior to anti-arrhythmic drug therapy for maintaining sinus rhythm and reducing the burden of $\mathrm{AF}^{39,43,48}$, and iv) class I and III anti-arrhythmic agents have been associated with increased all-cause mortality in several trials ${ }^{51-53}$. Theoretically, successful AF ablation could reduce total mortality by preventing thromboembolic events, heart failure decompensation and cardiovascular mortality, as suggested in the Framingham Heart Study ${ }^{47}$, or simply by allowing patients to stop their anti-arrhythmic drugs. However, data on this topic is contradictory. The Atrial Fibrillation and Congestive Heart Failure (AF-CHF) trial 
suggested that a routine strategy of rhythm control does not reduce the rate of death from cardiovascular causes in patients with atrial fibrillation and heart failure ${ }^{54}$. On the other hand, a sub-group analysis of the AFFIRM trial revealed that sinus rhythm was associated with lower mortality, although the main analysis had not shown any significant mortality benefit of a rhythm-control strategy, potentially attributed to antiarrhythmic drug adverse effects ${ }^{55}$. Similarly, a subgroup analysis of the RACE trial suggested that patients with mild to moderate chronic heart failure might fare better if successfully treated with a rhythm control strategy compared with rate control ${ }^{56}$.

However, whether ablation improves patients' outcomes with regards to adverse clinical endpoints such as death and stroke in all-comers remains to be unequivocally determined. Preliminary evidence favoring catheter ablation for the reduction of mortality is mostly restricted to the setting of heart failure and LV systolic dysfunction which our study now reinforces. In such patients, and especially in centers experienced in catheter ablation, it may be reasonable to prefer ablation over medical therapy alone with an aim to improve prognosis. On the other hand, the reduced stroke risk in observational data is not compelling enough, as observational data may suffer from the issue of the company which AF often keeps in the form of cardiovascular and other age-related comorbidity. Our analysis of observational data showed strong evidence of selection bias (e.g. differences in baseline characteristics between treatment groups, plus the pronounced reduction in stroke risk in observational studies which is not seen in randomized trials), despite attempts to limit such bias. An inherent selection bias in choosing "healthier" candidates for ablation can falsely give rise to better outcomes; a limitation which can be fully overcome only through rigorous randomization. The ongoing Catheter Ablation vs Anti-arrhythmic Drug Therapy for Atrial Fibrillation (CABANA) and Early Treatment of Atrial Fibrillation for Stroke Prevention (EAST) trials will provide much needed clarity in this area. 
In summary, at this time there is no compelling meta-analytic evidence for reduction in stroke risk after AF ablation, but randomized data are supportive of a survival benefit for AF ablation in heart failure patients with significant LV dysfunction. In this subgroup of patients, it is reasonable to consider catheter ablation as first-line treatment.

\section{Limitations}

The main limitation of this study is linked to its methodology and the heterogeneity between studies. Heterogeneity, assessed through the $\mathrm{I}^{2}$ test, was marked for the pooled analysis of allcause mortality and stroke. This was expected a priori given the methodological differences between observational studies. However, most importantly the sensitivity analysis of randomized studies showed no heterogeneity.

When interpreting the results of our sensitivity analysis of observational studies, the reader should be aware that, although the inclusion of data obtained through propensity-score or case-control matching reduces the degree of selection bias, it does not completely eliminate it. Patients receiving ablation in observational studies were older and had higher degree of comorbidity. Observational studies can only evaluate association, not causation. However, in our opinion this analysis is still worth reporting, for the reasons stated in the methods section.

Finally, the assessment of the benefit (or lack thereof) of catheter ablation for stroke reduction in randomized studies was underpowered due to the relatively low number of cerebrovascular events, while in observational studies the reader should take into consideration that reliable data on anticoagulation use was not consistently available.

\section{CONCLUSION}


In this meta-analysis, catheter ablation of atrial fibrillation was associated with a survival benefit compared with medical treatment alone; however, this was mainly noted in the setting of heart failure and left ventricular systolic dysfunction. Reduction in stroke risk was confined to observational studies alone. Further adequately powered randomized trials are needed to clarify whether ablation can be of benefit for stroke reduction as also for improving survival in populations other than those with LV dysfunction.

Acknowledgments: none

\section{REFERENCES}

1. Krijthe BP, Kunst A, Benjamin EJ et al. Projections on the number of individuals with atrial fibrillation in the European Union, from 2000 to 2060. Eur Heart J. 2013;34:2746-2751.

2. Ryder KM, Benjamin EJ. Epidemiology and significance of atrial fibrillation. Am $\mathbf{J}$ Cardiol 1999;84:131R-138R.

3. January CT, Wann LS, Alpert JS et al; ACC/AHA Task Force Members. 2014 AHA/ACC/HRS guideline for the management of patients with atrial fibrillation: executive summary: a report of the American College of Cardiology/American Heart Association Task Force on practice guidelines and the Heart Rhythm Society. Circulation. 2014 Dec 2;130(23):2071-104.

4. Hakalahti A, Biancari F, Nielsen JC, Raatikainen MJ. Radiofrequency ablation vs. antiarrhythmic drug therapy as first line treatment of symptomatic atrial fibrillation: systematic review and meta-analysis. Europace 2015;17:370-378.

5. Konstantinos C. Siontis, John P.A. Ioannidis, George D. Katritsis et al. Radiofrequency Ablation Versus Antiarrhythmic Drug Therapy for Atrial Fibrillation. JACC Clin Electrophysiol 2016;2:170-180.

6. Investigators TAFFI of RM (AFFIRM). A Comparison of Rate Control and Rhythm Control in Patients with Atrial Fibrillation. N Engl J Med 2002;347:1825-1833.

7. Steinberg JS, Sadaniantz A, Kron J et al. Analysis of cause-specific mortality in the Atrial Fibrillation Follow-up Investigation of Rhythm Management (AFFIRM) study. Circulation. 2004 Apr 27;109(16):1973-80. 
8. Noseworthy PA, Kapa S, Deshmukh AJ et al. Risk of stroke after catheter ablation versus cardioversion for atrial fibrillation: A propensity-matched study of 24,244 patients. Hear Rhythm 2015;12:1154-1161.

9. Stabile G, Bertaglia E, Senatore G et al. Catheter ablation treatment in patients with drug-refractory atrial fibrillation: A prospective, multi-centre, randomized, controlled study (Catheter Ablation for the Cure of Atrial Fibrillation Study). Eur Heart J. 2006;27:216-221.

10. Chang $\mathrm{CH}$, Lin JW, Chiu FC et al. Effect of radiofrequency catheter ablation for atrial fibrillation on morbidity and mortality: a nationwide cohort study and propensity score analysis. Circ Arrhythm Electrophysiol. 2014;7:76-82.

11. Friberg L, Tabrizi F, Englund A. Catheter ablation for atrial fibrillation is associated with lower incidence of stroke and death: data from Swedish health registries. Eur Heart J 2016;37:1825-1833.

12. Di Biase L, Mohanty P, Mohanty S et al. Ablation Versus Amiodarone for Treatment of Persistent Atrial Fibrillation in Patients With Congestive Heart Failure and an Implanted Device: Results From the AATAC Multicenter Randomized Trial. Circulation. 2016;133:1637-1644.

13. Hummel J, Michaud G, Hoyt R et al; TTOP-AF Investigators. Phased RF ablation in persistent atrial fibrillation. Hear Rhythm 2014;11:202-209.

14. Packer DL, Kowal RC, Wheelan KR et al; STOP AFCryoablation Investigators. Cryoballoon ablation of pulmonary veins for paroxysmal atrial fibrillation: First results of the North American arctic front (STOP AF) pivotal trial. J Am Coll Cardiol 2013;61:1713-1723.

15. Morillo CA, Verma A, Connolly SJ et al; RAAFT-2 Investigators. Radiofrequency ablation vs antiarrhythmic drugs as first-line treatment of paroxysmal atrial fibrillation (RAAFT-2): a randomized trial. Jama 2014;311:692-700.

16. Menzies D. Systematic reviews and meta-analyses. Int J Tuberc Lung Dis 2011;15:582-93.

17. Moher D, Liberati A, Tetzlaff J et al. Preferred reporting items for systematic reviews and meta-analyses: the PRISMA statement. Ann Intern Med 2009;151:264-9, W64.

18. Verhagen A, de Vet HC, de Bie RA et al. The Delphi list: a criteria list for quality assessment of randomized clinical trials for conducting systematic reviews developed by Delphi consensus. J Clin Epidemiol 1998;51:1235-41.

19. Ottawa Hospital Research Institute; Available from: http://www.ohri.ca/programs/clinical_epidemiology/oxford.asp 
20. Bunch TJ, Crandall BG, Weiss JP et al. Patients treated with catheter ablation for atrial fibrillation have long-term rates of death, stroke, and dementia similar to patients without atrial fibrillation. J Cardiovasc Electrophysiol. 2011;22:839-845.

21. Lin YJ, Chao TF, Tsao HM et al. Successful catheter ablation reduces the risk of cardiovascular events in atrial fibrillation patients with CHA2DS2-VASc risk score of 1 and higher. Europace. 2013;15:676-684.

22. Pappone C, Rosanio S, Augello G et al. Mortality, morbidity, and quality of life after circumferential pulmonary vein ablation for atrial fibrillation: Outcomes from a controlled nonrandomized long-term study. J Am Coll Cardiol. 2003;42:185-197.

23. Saliba W, Schliamser JE, Lavi I, Barnett-Griness O, Gronich N, Rennert G. Catheter ablation of atrial fibrillation is associated with reduced risk of stroke and mortality: A propensity score-matched analysis. Hear Rhythm 2017 May;14(5):635-642.

24. Hunter RJ, McCready J, Diab I et al. Maintenance of sinus rhythm with an ablation strategy in patients with atrial fibrillation is associated with a lower risk of stroke and death. Heart. 2012;98:48-53.

25. Hunter RJ, Berriman TJ, Diab I et al.A randomized controlled trial of catheter ablation versus medical treatment of atrial fibrillation in heart failure (the CAMTAF trial). Circ Arrhythmia Electrophysiol. 2014;7:31-38.

26. Blandino A, Toso E, Scaglione M et al. Long-term efficacy and safety of two different rhythm control strategies in elderly patients with symptomatic persistent atrial fibrillation. J Cardiovasc Electrophysiol. 2013;24:731-738.

27. Reynolds MR, Gunnarsson CL, Hunter TD et al. Health outcomes with catheter ablation or antiarrhythmic drug therapy in atrial fibrillation results of a propensitymatched analysis. Circ Cardiovasc Qual Outcomes. 2012;5:171-181.

28. MacDonald MR, Connelly DT, Hawkins NM et al. Radiofrequency ablation for persistent atrial fibrillation in patients with advanced heart failure and severe left ventricular systolic dysfunction: a randomised controlled trial. Heart 2011;97:740-747.

29. Cosedis Nielsen J, Johannessen A, Raatikainen P et al. Radiofrequency Ablation as Initial Therapy in Paroxysmal Atrial Fibrillation. Nejm. 2012;367(17):1587-95.

30. Pappone C, Vicedomini G, Augello G, et al. Radiofrequency catheter ablation and antiarrhythmic drug therapy: A prospective, randomized, 4-year follow-up trial: The APAF study. Circ Arrhythmia Electrophysiol. 2011;4:808-814.

31. Krittayaphong R, Raungrattanaamporn O, Bhuripanyo K et al. A randomized clinical trial of the efficacy of radiofrequency catheter ablation and amiodarone in the treatment of symptomatic atrial fibrillation. J Med Assoc Thai 2003;86 Suppl 1:S8-16. 
32. Forleo GB, Mantica M, De Luca L et al. Catheter ablation of atrial fibrillation in patients with diabetes mellitus type 2 : Results from a randomized study comparing pulmonary vein isolation versus antiarrhythmic drug therapy. J Cardiovasc Electrophysiol. 2009;20:22-28.

33. Jaïs P, Cauchemez B, Macle L et al. Catheter ablation versus antiarrhythmic drugs for atrial fibrillation: The A4 study. Circulation. 2008;118:2498-2505.

34. Mont L, Bisbal F, Hernández-Madrid A et al; SARA investigators. Catheter ablation vs. antiarrhythmic drug treatment of persistent atrial fibrillation: A multicentre, randomized, controlled trial (SARA study). Eur Heart J. 2014;35:501-507.

35. Om W, Nf M, Do M et al. Apples and Oranges Comparing Antiarrhythmic Drugs and Catheter Ablation for Treatment of Atrial Fibrillation. Circ Arrhythmia Electrophysiol 2014;7:853-860.

36. Wilber DJ, Pappone C, Neuzil P et al; ThermoCool AF Trial Investigators. Comparison of antiarrhythmic drug therapy and radiofrequency catheter ablation in patients with paroxysmal atrial fibrillation: a randomized controlled trial. JAMA. 2010 Jan 27;303(4):333-40.

37. Oral H, Pappone C, Chugh A et al. Circumferential Pulmonary-Vein Ablation for Chronic Atrial Fibrillation. 2006;934-941.

38. Khan MN, Jaïs P, Cummings $\mathbf{J}$ et al; PABA-CHF Investigators. Pulmonary-Vein Isolation for Atrial Fibrillation in Patients with Heart Failure. N Engl J Med 2008;359:1778-1785.

39. Choi AD, Hematpour K, Kukin M, Mittal S, Steinberg JS. Ablation vs medical therapy in the setting of symptomatic atrial fibrillation and left ventricular dysfunction.

Congest Hear Fail. 2010;16:10-14.

40. Jones DG, Haldar SK, Hussain W et al. A randomized trial to assess catheter ablation versus rate control in the management of persistent atrial fibrillation in heart failure. $\mathrm{J}$ Am Coll Cardiol 2013;61:1894-1903.

41. Prabhu S, Taylor AJ, Costello BT et al. Catheter Ablation Versus Medical Rate control in Atrial Fibrillation and Systolic Dysfunction (CAMERA-MRI), Journal of the American College of Cardiology (2017), doi: 10.1016/j.jacc.2017.08.041

42. Marrouche N, Brachmann J, Andresen D et al. Catheter Ablation for Atrial Fibrillation with Heart Failure. N Engl J Med 2018; 378:417-427. DOI: 10.1056/NEJMoa1707855

43. Gentlesk PJ, Sauer WH, Gerstenfeld EP et al. Reversal of left ventricular dysfunction following ablation of atrial fibrillation. J Cardiovasc Electrophysiol 2007;18:9-14. 
44. Al Halabi S, Qintar M, Hussein A et al. Catheter Ablation for Atrial Fibrillation in Heart Failure Patients: A Meta-Analysis of Randomized Controlled Trials. JACC Clin Electrophysiol 2015;1:200-209.

45. Nedios S, Sommer P, Dagres $\mathrm{N}$ et al. Long-term follow-up after atrial fibrillation ablation in patients with impaired left ventricular systolic function: The importance of rhythm and rate control. Hear Rhythm 2014;11:344-351.

46. Natale A, Thakur RK. Cardioembolic stroke. Cardiol Clin. 2016 May;34(2):xiii-xiv.

47. Ghanbari H, Başer K, Jongnarangsin K et al. Mortality and cerebrovascular events after radiofrequency catheter ablation of atrial fibrillation. Hear Rhythm 2014;11:1503-1511.

48. Chen MS, Marrouche NF, Khaykin Y et al. Pulmonary vein isolation for the treatment of atrial fibrillation in patients with impaired systolic function. J Am Coll Cardiol 2004;43:1004-1009.

49. Benjamin EJ, Wolf PA, D'Agostino RB, Silbershatz H, Kannel WB, Levy D. Impact of atrial fibrillation on the risk of death: the Framingham Heart Study. Circulation 1998;98:946-52.

50. Glotzer TV, Daoud EG, Wyse DG et al. The Relationship Between Daily Atrial Tachyarrhythmia Burden From Implantable Device Diagnostics and Stroke Risk: The TRENDS study. Circ Arrhythmia Electrophysiol 2009;2(5):474-80.

51. Køber L, Torp-Pedersen C, McMurray JJ et al; Dronedarone Study Group. Increased Mortality after Dronedarone Therapy for Severe Heart Failure. N Engl J Med 2008;358:2678-2687.

52. Echt DS, Liebson PR, Mitchell LB et al. Mortality and Morbidity in Patients Receiving Encainide, Flecainide, or Placebo. N Engl J Med 1991;324:781-788.

53. Connolly SJ, Camm AJ, Halperin JL et al; PALLAS Investigators. Dronedarone in High-Risk Permanent Atrial Fibrillation. N Engl J Med 2011;365:2268-2276.

54. Roy D, Talajic M, Nattel S et al; Atrial Fibrillation and Congestive Heart Failure Investigators. Rhythm control versus rate control for atrial fibrillation and heart failure. N Engl J Med. 2008 Jun 19;358(25):2667-77. doi: 10.1056/NEJMoa0708789.

55. Wyse DG, Waldo AL, DiMarco JP et al; Atrial Fibrillation Followup Investigation of Rhythm Management (AFFIRM) Investigators. A Comparison of Rate Control and Rhythm Control in Patients with Atrial Fibrillation. N Engl J Med 2002;347:1825-1833.

56. Hagens VE, Crijns HJ, Van Veldhuisen DJ et al; RAteControl versus Electrical cardioversion for persistent atrial fibrillation study gr 
oup. Rate control versus rhythm control for patients with persistent atrial fibrillation with mild to moderate heart failure: Results from the RAte Control versus Electrical cardioversion (RACE) study. Am Heart J 2005;149:1106-1111.

\section{FIGURE LEGENDS}

Figure 1 - Primary endpoint: All-cause mortality. Forest plots comparing ablation vs. no ablation groups separately in randomized and observational studies

Figure 2 - Secondary endpoint: Cerebrovascular event. Forest plots comparing ablation vs. no ablation groups separately in randomized and observational studies 
TABLE 1 - Selected studies for the systematic review

\begin{tabular}{|c|c|c|c|c|c|}
\hline \multirow{2}{*}{ Author, ref } & \multirow{2}{*}{ Study design } & \multicolumn{3}{|c|}{ Sample size (patients) } & \multirow{2}{*}{$\begin{array}{l}\text { Mean follow-up } \\
\text { (months) }\end{array}$} \\
\hline & & Total & Ablation & No ablation & \\
\hline Krittayaphong et al 2003 & RCT single-center & 30 & 15 & 15 & 12 \\
\hline Pappone et al 2003 & Observational single-centre & 1171 & 589 & 582 & 29.6 \\
\hline Wazni et al 2005 & RCT multi-centre & 70 & 33 & 37 & 12 \\
\hline Stabile et al 2006 & RCT multi-centre & 137 & 68 & 69 & 12 \\
\hline Oral et al 2006 & RCT multi-centre & 146 & 77 & 69 & 12 \\
\hline Forleo et al 2009 & RCT multi-centre & 70 & 35 & 35 & 12 \\
\hline Jais et al 2008 & RCT multi-centre & 112 & 53 & 59 & 12 \\
\hline Wilber et al 2010 & RCT multi-centre & 167 & 106 & 61 & 9 \\
\hline Choi et al 2010 & Observational single-centre & 30 & 15 & 15 & 16 \\
\hline Hunter et al 2011 & Observational multi-centre & 5465 & 1273 & 4192 & 17.8 \\
\hline Bunch et al 2011 & Observational multi-centre & 21060 & 4212 & 16848 & 36 \\
\hline Pappone et al 2011 & RCT single-centre & 198 & 99 & 99 & 48 \\
\hline MacDonald et al 2011 & RCT multi-centre & 41 & 22 & 19 & 6 \\
\hline Cosedis Nielsen et al 2012* & RCT multi-centre & 294 & 146 & 148 & 24 \\
\hline Reynolds et al 2012 & Observational multi-centre & 1602 & 801 & 801 & 27 \\
\hline Lin et al 2013 & Observational single-centre & 348 & 174 & 174 & 47 \\
\hline Jones et al 2013 & RCT single-centre & 52 & 26 & 26 & 12 \\
\hline Blandino et al 2013 & Observational single-centre & 422 & 153 & 269 & 60 \\
\hline Packer et al 2013 & RCT multi-centre & 245 & 163 & 82 & 12 \\
\hline Mont et al 2014 & RCT multi-centre & 146 & 98 & 48 & 12 \\
\hline Morillo et al 2014 & RCT multi-centre & 127 & 66 & 61 & 21 \\
\hline Chang et al 2014 & Observational multi-centre & 12170 & 846 & 11324 & 47.3 \\
\hline Hummel et al 2014 & RCT multi-centre & 204 & 132 & 72 & 6 \\
\hline Hunter et al 2014 & RCT single-centre & 50 & 26 & 24 & 6 \\
\hline Noseworthy et al 2015 & Observational multi-centre & 24244 & 12122 & 12122 & 28.8 \\
\hline Di Biase et al 2016 & RCT multi-centre & 203 & 102 & 101 & 24 \\
\hline
\end{tabular}




\begin{tabular}{llcccc}
\hline Friberg et al $\mathbf{2 0 1 6}$ & Observational multi-centre & 4992 & 2496 & 2496 & 52.8 \\
\hline Saliba et al $\mathbf{2 0 1 7}$ & Observational multi-centre & 4741 & 969 & 3772 & 48 \\
\hline Marrouche et al 2017 & RCT multi-centre & 363 & 179 & 184 & 37.8 \\
\hline Prabhu et al 2017 & RCT multi-centre & 66 & 33 & 33 & 6 \\
\hline
\end{tabular}

Legends: RCT- Randomized controlled trial *A five-year follow-up study was published in March 2017, yet only 245 of the initial 294 patients completed such follow-up.

TABLE 2 - Overall baseline characteristics of atrial fibrillation patients receiving catheter ablation vs. no ablation in randomized trials

\begin{tabular}{|c|c|c|}
\hline & \multicolumn{2}{|c|}{ Baseline characteristics } \\
\hline & Ablation & No ablation \\
\hline Age (years, mean) & 58 & 58.2 \\
\hline Male gender (\%) & 75.3 & 75.5 \\
\hline Paroxysmal AF (\%) & 49.7 & 49.6 \\
\hline Time since AF diagnosis (years, mean) & 2.9 & 2.9 \\
\hline On antiarrhythmic drugs (\%) & 63.5 & 72.2 \\
\hline On anticoagulation * & 100 & 100 \\
\hline History of hypertension (\%) & 45.4 & 46.2 \\
\hline History of Diabetes Mellitus (\%) & 9.6 & 10.4 \\
\hline History of CAD/Myocardial infarction (\%) & 14.2 & 13.9 \\
\hline History of cerebrovascular event (\%) & 3 & 3.5 \\
\hline NYHA class $\geq 2(\%)$ & 34 & 34.5 \\
\hline LV ejection fraction (\%, mean) & 50.6 & 51 \\
\hline Left atrium diameter (cm, mean) & 42.6 & 40 \\
\hline
\end{tabular}

Legends: AF- Atrial fibrillation; CAD- Coronary artery disease; LV- Left ventricular; NYHA- New York Heart Association

* Contraindication for anticoagulation was an exclusion criteria in randomized studies 